\title{
Favorable long-term outcomes of bilateral adrenalectomy in Cushing's disease
}

\author{
Andrea Oßwald ${ }^{1}$, Eva Plomer', Christina Dimopoulou',2, Monika Milian³, \\ Rainer Blaser ${ }^{4}$, Katrin Ritzel ${ }^{1}$, Anne Mickisch ${ }^{1}$, Ferengis Knerr ${ }^{1}$, Milan Stanojevic ${ }^{3}$, \\ Klaus Hallfeldt' ${ }^{5}$, Jochen Schopohl' ${ }^{1}$, Klaus A Kuhn ${ }^{4}$, Günter Stalla ${ }^{2}$, Felix Beuschlein ${ }^{1}$ \\ and Martin Reincke ${ }^{1}$
}

${ }^{1}$ Medizinische Klinik und Poliklinik IV, Ludwig-Maximilians-Universität München, Klinikum der Universität München, Ziemssenstraße 1, D-80336 Munich, Germany, 2Department of Endocrinology, Max Planck Institute of Psychiatry, München, Germany, ${ }^{3}$ Department of Neurosurgery, University Hospital Tuebingen, Tübingen, Germany, ${ }^{4}$ IMSE, Klinikum rechts der Isar der TU München, München, Germany and ${ }^{5}$ Chirurgische Klinik und Poliklinik Innenstadt, Klinikum der Ludwig-Maximilians-Universität München, München, Germany
Correspondence should be addressed to M Reincke

Email martin.reincke@med. uni-muenchen.de

\begin{abstract}
Objective: Bilateral adrenalectomy (BADX) is an important treatment option for patients with Cushing's syndrome (CS). Our aim is to analyze the long-term outcomes, surgical, biochemical, and clinical as well as morbidity and mortality, of patients who underwent BADX.

Design: A total of 50 patients who underwent BADX since 1990 in two German centers were identified. Of them, 34 patients had Cushing's disease (CD), nine ectopic CS (ECS), and seven ACTH-independent bilateral adrenal hyperplasia (BAH).

Methods: Standardized follow-up examination was performed in 36 patients with a minimum follow-up time of 6 months after BADX and a median follow-up time of 11 years.

Results: Surgical morbidity and mortality were 6 and $4 \%$ respectively. All patients were found to be in remission after BADX. Almost all Cushing's-specific comorbidities except for psychiatric diseases improved significantly. Health-related quality of life remained impaired in $45.0 \%$ of female and $16.7 \%$ of male patients compared with a healthy population. The median number of adrenal crises per 100 patient-years was four. Nelson tumor occurred in $24 \%$ of CD patients after a median time span of 51 months. Long-term mortality after 10 years was high in ECS (44\%) compared with CD (3\%) and BAH (14\%).

Conclusions: BADX is an effective and relatively safe treatment option especially in patients with $C D$. The majority of patients experience considerable improvement of Cushing's symptoms.
\end{abstract}

\section{Introduction}

Endogenous Cushing's syndrome (CS) is a severe disease induced by excess glucocorticoid production. CS is associated with an increased cardiovascular risk, psychological side effects, musculoskeletal impairment, and decreased quality of life (1). In more than $80 \%$ of the cases, hypercortisolism is adrenocorticotropin (ACTH) dependent. The sources of ACTH production are predominantly ACTH-secreting pituitary adenomas (Cushing's disease, CD) and less frequently ectopic, non-pituitary neuroendocrine tumors (ectopic CS, ECS).
ACTH-independent CS results from autonomous cortisol production in the adrenal cortex. It is mostly caused by unilateral adrenal adenomas and less frequently by cortisolsecreting carcinomas and bilateral adrenal disorders (2).

First-line therapeutic approach to $\mathrm{CD}$ is a transsphenoidal surgery (TSS) with removal of the ACTHproducing adenoma. The remission rate is reported to range from 69 to $82 \%$ with a recurrence rate even after initial normalization of hypercortisolism of $9-17 \%(3,4)$. Treatment options for $\mathrm{CD}$ after a failed initial therapy or
(ㄷ) 2014 European Society of Endocrinology Printed in Great Britain 
recurrence include repeated TSSs (5), radiotherapy $(6,7)$, medical treatment (8), and bilateral adrenalectomy (BADX). As reflected in an Italian multicenter study with almost $10 \%$ of CD patients who underwent BADX (4), this procedure is necessary in a relevant subgroup of patients. In case of ECS, first-line treatment is the removal of ACTHproducing tumor. BADX is the preferred option when the source of ectopic ACTH production is undetectable or cannot be removed. Another indication for BADX is bilateral ACTH-independent adrenal hyperplasia (BAH).

Although BADX has been implemented in clinical praxis over a long period of time, the outcome of patients has mostly been studied retrospectively in case reports or small series. Therefore, our goal is to analyze the long-term outcomes, surgical, biochemical, and clinical as well as morbidity and mortality of all BADX patients treated in two institutions since 1990 in a standardized way.

\section{Patients and methods}

\section{Patient cohort}

Patients with CS treated in two Munich centers of the German Cushing's Registry CUSTODES (Cushing's Syndrome - Therapie und Outcome in Deutschland) from 1990 to 2013 were identified. Out of 254 patients of the Medizinische Klinik und Poliklinik IV, 45 underwent BADX and out of 58 patients of the MaxPlanck-Institut für Psychiatrie, five underwent BADX.

Epidemiological, clinical, and biochemical data were extracted retrospectively from patient's files and entered into an electronic database. All surviving patients with minimum follow-up time of 6 months after BADX $(n=40)$ were contacted by mail and asked to participate in a prospective long-term evaluation of CS within the frame of the German Cushing's Registry CUSTODES. Of them, 36 patients agreed to participate and four patients were lost to follow-up. Eight patients did not present in person, but were interviewed by telephone and medical records were obtained. Herein, we report on the data of the first annual survey conducted in 2013 in a cross-sectional manner. The ethics committee of the participating centers approved the protocol of the German Cushing's Registry and all patients provided written informed consent.

Information on medical history and sociodemographic characteristics was obtained by trained and certified staff members during a personal interview. Moreover, the participants underwent an extensive standardized medical examination, including blood sampling, evaluation of waist-to-hip ratio, grip strength, body composition, and measurement of systolic and diastolic blood pressure. Body composition was determined by a body impedance analyzer BIA 2000-S (Data Input). Systolic and diastolic blood pressures were measured three times on both arms in seated position. The mean of the three measurements was used. Grip strength was assessed using the Jamar hydraulic hand dynamometer (Sammons Preston Rolyan, Bolingbrook, IL, USA). The mean values of three trials of the leading hand were calculated and compared with the median values of an age- and gender-matched normative population (9). Evaluation of the past medical history included past and current signs and symptoms of CS, intermittent remission and recurrences of CS, therapy, cardiovascular events, psychiatric comorbidities, metabolic comorbidities, and musculoskeletal comorbidities. For the comparison of values and comorbidities before and after BADX, parameters before BADX were assessed at the visit immediately before the first treatment. Then, BADX parameters were assessed by the standardized follow-up cross-sectional study.

Postoperatively, glucocorticoids were substituted mostly with hydrocortisone (median dose, $25 \mathrm{mg}$; range, 15-45 mg). In four patients, glucocorticoids were substituted with modified-release hydrocortisone (median dose, $20 \mathrm{mg}$; range, 20-40 mg). Mineralocorticoid substitution was performed with fludrocortisone (median dose, $0.05 \mathrm{mg}$; range, $0.025-0.20 \mathrm{mg}$ ). In five women, DHEA was used for substitution.

\section{Biochemical measurements}

Blood samples were taken in the morning from fasting, seated subjects without hydrocortisone whenever possible. Free urine cortisol was assayed by the Centaur Immunoassay, Siemens (Erlangen, Germany). ACTH and serum cortisol were measured by the Liaison Assay, DiaSorin (Saluggia, Italy). Conversion from ACTH in $\mathrm{pg} / \mathrm{ml}$ to SI unit pmol/l is performed by multiplying by 0.22 . All other biochemical variables were assayed in our central laboratory using standard methods.

\section{Endocrine assessment of remission of CS}

Remission from CS was defined by dependence on hydrocortisone substitution.

\section{Definitions}

Subjects with blood pressures $\geq 140 / 90 \mathrm{mmHg}$ or a selfreported history of hypertension and intake of antihypertensive medication were considered hypertensive. Diabetes was defined by $\mathrm{HbA} 1 \mathrm{c} \geq 6.5 \%$ or intake of antidiabetic 
drugs. Osteopenia was defined by $T$-values $\leq-1.0$ S.D. and osteoporosis by $T$-values $\leq-2.5$ S.D., using dual energy $\mathrm{X}$-ray absorptiometry (DEX). Cushing's stigmata included moon face, buffalo hump, hirsutism, or skin alterations. Muscle weakness was defined by the disability to rise from knee bending without the aid of arms. Psychiatric morbidities included self-reported anxiety, depression, panic attacks, or psychosis. The presence of adrenal remnants was defined on the basis of radiological or biochemical evidence. Adrenal crisis was defined as an acute impairment of general health that necessitated i.v. glucocorticoid administration and hospital admission. The presence of Nelson tumor was defined by the growth of an adenoma that had led to additional treatments (TSS and radiotherapy) in patients with CD following BADX. Surgical mortality and morbidity were defined as any death or surgery-related morbidity occurring in between 30 days after surgery.

\section{Health-related quality of life questionnaires}

A generic health-related quality of life (HRQoL) questionnaire (SF-36 Health Survey) (10) and a disease-specific instrument (Tuebingen CD-25) (11) were used for the assessment of HRQoL. Age- and gender-specific normative data were available for both instruments.

\section{Statistical analysis}

The data were extracted from the German Cushing's Registry CUSTODES. Results are expressed as median and interquartile range (IQR) or mean and s.D. Data before and after BADX were compared using the $\chi^{2}$-test (two-sided) or the two-sided Wilcoxon signed-rank test. Concerning HRQoL, patients were allocated to the categories impaired vs not impaired according to the age- and gender-specific normative data of both instruments, and thus, the percentage of impaired patients is shown. Additionally, independent samples $t$-tests were used for the comparison of the Tuebingen CD-25 Total Score between BADX patients and a group of patients with florid CD. $P<0.05$ was considered statistically significant. Statistical analysis was performed using the standard statistical software (SPSS 21).

\section{Results}

A total of 50 patients with CS who underwent BADX since 1990 were identified. Figure 1 summarizes the etiology of CS of this patient cohort.

The median duration of CS from diagnosis to BADX was 44 months for CD (IQR 75), 4 months for ECS

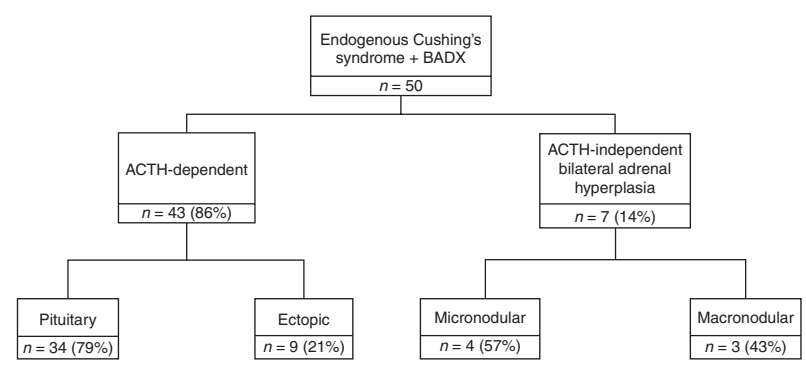

\section{Figure 1}

Etiology of Cushing's syndrome for all patients with bilateral adrenalectomy (BADX) treated in two Munich centers from 1990 to 2013.

(IQR 31), and 1 month for BAH (IQR 1). In all BAH and two occult ectopic cases, BADX was performed as first-line treatment. Five patients with $\mathrm{CD}$ underwent BADX as first-line treatment in the 1960s-1970s when this was the only therapeutic approach. The remaining CD patients underwent BADX after one (62\%) or more (38\%) TSSs. Of the total number of CD patients, 21 and $45 \%$ received at least one radiation or medical therapy respectively. Of the remaining ECS patients, 29\% received medical treatments, $29 \%$ underwent an operation of the ectopic source of ACTH production, and $43 \%$ received both treatment options before BADX.

\section{Surgical outcome}

BADX was performed laparoscopically in 34 cases (68\%) and by an open approach in 15 cases (30\%). The cases with open approach were all performed before laparoscopic techniques were available. In one case, conversion to open BADX was necessary. Two patients (4\%) died within the first 30 days after BADX (Table 1). As surgical morbidity, infections $(n=2)$ and thromboembolism $(n=1)$ were reported.

\section{Long-term morbidity}

Out of 35 patients, 22 (63\%) experienced at least one adrenal crisis, defined by the need for i.v. glucocorticoid administration. The median number of adrenal crises (one or more) per 100 patient-years was 4.1 (IQR 31). Nelson tumor occurred in $24 \%$ (7/29) of the patients with CD and BADX as second-line treatment. All of these patients required radiation and three needed TSS too. Two patients developed Nelson tumors after gamma knife resection. The median ACTH levels in patients with Nelson tumors were $350 \mathrm{pg} / \mathrm{ml}$. 
Table 1 Etiology of Cushing's syndrome (CS) and cause of death.

\begin{tabular}{|c|c|c|c|}
\hline Patient & $\begin{array}{l}\text { Cushing's } \\
\text { subtype }\end{array}$ & Cause of death & $\begin{array}{l}\text { Surgery- } \\
\text { related death }\end{array}$ \\
\hline 1 & $C D$ & Renal failure & $x$ \\
\hline 2 & $C D$ & Shock of unknown origin & \\
\hline 3 & BAH & Postoperative bleeding & $x$ \\
\hline 4 & ECS & $\begin{array}{l}\text { Stomach perforation } \\
\text { by tumor }\end{array}$ & \\
\hline 5 & ECS & $\begin{array}{l}\text { Hepatorenal failure } \\
\text { through metastatic } \\
\text { disease }\end{array}$ & \\
\hline 6 & ECS & Cardiogenic shock & \\
\hline 7 & ECS & Sepsis with adrenal crisis & \\
\hline
\end{tabular}

CD, Cushing's disease; BAH, bilateral adrenal hyperplasia; ECS, ectopic Cushing's syndrome.

\section{Mortality}

Total mortality was 14\%. The Kaplan-Meier curve (Fig. 2) shows subtype-specific survival that was excellent in CD (mortality at $1,2,5$, and 10 years was $2.9 \%$ ). In $\mathrm{BAH}$, mortality was $14 \%$ at $1,2,5$, and 10 years. Mortality was highest in ECS (22\% at 1 year and $44 \%$ at 2, 5, and 10 years). In addition to the two BADX-related cases of death, mortality was due to a cardiogenic shock $(n=1)$, a shock of unknown origin $(n=1)$, tumor-related death in ECS cases $(n=2$; hepatorenal failure through metastasis and stomach perforation by tumor), and sepsis with adrenal crisis $(n=1)$. The mean time between BADX and death were 2.6 years. The mean age at death was 60 years.

\section{Biochemical outcome}

All 36 patients evaluated were in remission from CS and none showed a relapse. Adrenal remnants remained in three patients necessitating adrenal revision in one case. ACTH values increased from a median of $54 \mathrm{pg} / \mathrm{ml}$ (IQR $63 \mathrm{pg} / \mathrm{ml}$ ) to $203 \mathrm{pg} / \mathrm{ml}$ (IQR $313 \mathrm{pg} / \mathrm{ml}$ ) after BADX in CD patients without Nelson tumors $(n=6, P=0.046)$.

\section{Clinical outcome}

Standardized follow-up examination was performed in 36 patients with a median follow-up time of 11 years since BADX (range 0.8-51 years). Table 2 shows the improvement of Cushing's-specific comorbidities and cardiovascular risk factors after BADX. Table 3 shows clinical and biochemical outcomes at follow-up examination according to the length of follow-up since BADX divided into tertiles with median follow-up times of 3,10 , and 36 years. The rate of impairment in HRQoL in patients with CS after BADX and patients with florid CD compared with a healthy population is shown in Table 4. A lower rate of impaired patients refers to a better quality of life. Quality of life remained impaired in $45.0 \%$ of female patients and $16.7 \%$ of the male patients with CS after BADX compared with a healthy population. However, compared with a cohort of patients with untreated florid CD, patients who underwent BADX showed better disease-specific HRQoL (12). In Table 5, the hydrocortisone maintenance dose is correlated with the persistence of morbidities after BADX.

\section{Discussion}

While BADX remains an important treatment option in patients with CS, only a few studies have analyzed the long-term outcomes after BADX systematically. Most of the studies were retrospective cohort studies with small numbers of patients and relatively short length of followup. To our knowledge this is one of the biggest studies combined with one of the longest follow-up periods in the literature. The data demonstrate that BADX is an excellent treatment option for all patients with CS regardless of the underlying etiology, but that long-term outcomes are especially favorable in CD.

Surgical mortality and morbidity with 4 and 6\% were comparable to published data showing a median of 3 and

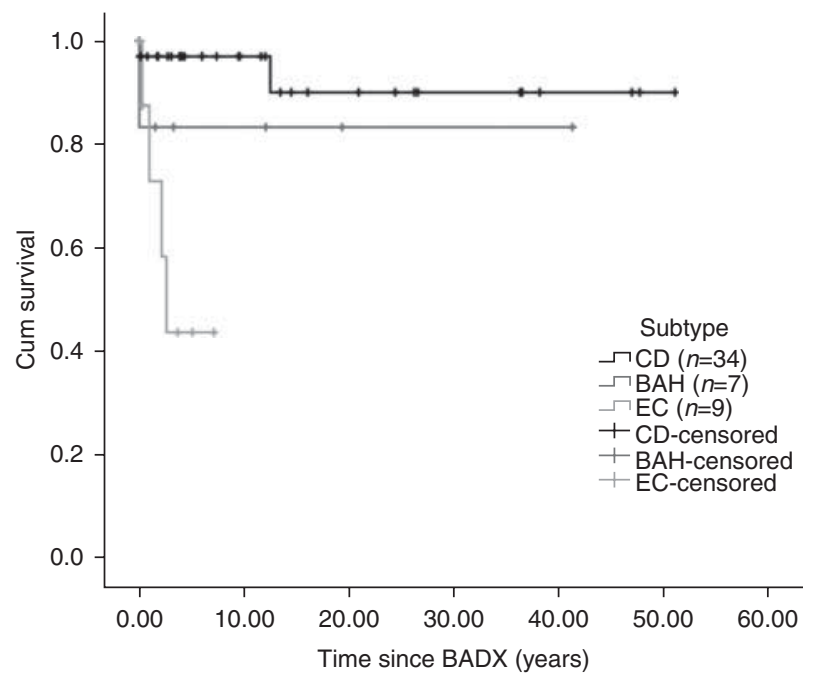

\section{Figure 2}

Survival in patients with Cushing's syndrome according to Cushing subtype (Kaplan-Meier method). CD, Cushing's disease; $\mathrm{BAH}$, bilateral adrenal hyperplasia; ECS, ectopic Cushing's syndrome. 
Table 2 Comorbidities and cardiovascular risk factors in patients with CS before and after bilateral adrenalectomy (BADX) obtained by standardized follow-up examination.

\begin{tabular}{|c|c|c|c|}
\hline & $\begin{array}{c}\text { Before BADX } \\
n(\%)\end{array}$ & $\begin{array}{c}\text { After BADX } \\
n(\%)\end{array}$ & $\boldsymbol{P}$ \\
\hline High blood pressure & $31(91)$ & $25(69)$ & 0.023 \\
\hline Diabetes mellitus & $14(45)$ & $6(17)$ & 0.011 \\
\hline $\begin{array}{l}\text { Osteopenia or } \\
\text { osteoporosis }\end{array}$ & $27(84)$ & $30(73)$ & 0.251 \\
\hline Cushing's stigmata & $34(100)$ & $8(22)$ & 0.000 \\
\hline Muscle weakness & $21(72)$ & $11(33)$ & 0.002 \\
\hline Psychiatric morbidity & $16(53)$ & $17(47)$ & 0.621 \\
\hline Menstrual irregularity ${ }^{a}$ & $14(82)$ & $4(50)$ & 0.093 \\
\hline
\end{tabular}

${ }^{a}$ Excluding postmenopausal women.

$18 \%$ respectively (13). As demonstrated earlier, the laparoscopic approach was not superior to open BADX regarding mortality and morbidity (14). With no surgery-related death in patients with ECS, we could not confirm an enhanced surgical mortality in this subgroup (13). Long-term mortality was favorably low in CD and significantly higher in ECS compared with the other subtypes in accordance with published data (13). Remarkably, after 25 years, 94\% of patients with $\mathrm{CD}$ were still alive. In accordance with the observation that death in the first 12 months is particularly high, $40 \%$ of the non-surgical-related deaths occurred within the first 12 months after BADX (15).

BADX was an effective method resulting in remission of hypercortisolism in all patients. Revision due to adrenal remnants was necessary in one patient (2\%). ACTH levels increased after BADX in patients having $\mathrm{CD}$ without Nelson tumors as reported previously (16).

According to our standardized examination, improvement of nearly all Cushing's-related comorbidities such as hypertension, diabetes, osteoporosis, and muscle weakness was evident after a median follow-up time of 11 years. Notably, menstrual irregularity and psychiatric morbidity did not change distinctively which is in accordance with earlier studies (17). Similarly, and as shown before, some Cushing's stigmata such as full moon face, hirsutism, and parchment skin also remain even after a long follow-up period (18). It has also been demonstrated that elevated cardiovascular risk factors persist after a successful TSS in CD patients (19). Therefore, these comorbidities might rather depend on the impact of former hypercortisolism than on the treatment option.

Interestingly, improvement of comorbidities was partly depending on the length of follow-up since BADX: blood pressure, HbA1c, and $T$-value in DEX were normalized within the first 5 years after BADX. However, during long-term follow-up, high blood pressure and lower bone density re-appeared, most probably in an age-dependent manner. Notably, the incidence of diabetes mellitus appears to be stable during long-term follow-up. The BMI and waist-to-hip ratio were comparable to German cohorts during all follow-up times (20). The median grip strength reached $83-90 \%$ of the median of an age- and gender-matched cohort.

General health-related and Cushing's-specific quality of life remained more frequently impaired in female BADX

Table 3 Clinical and biochemical outcomes in patients with CS after BADX according to the duration of follow-up since BADX divided into tertiles.

Females (\%)
Follow-up as BADX (years)
Age at follow-up (years)
Systolic blood pressure $(\mathrm{mmHg})$
Diastolic blood pressure $(\mathrm{mmHg})$
HbA1c $(\%)$
Cholesterol (mg/dl)
LDL (mg/dl)
Triglycerides (mg/dl)
$T$-value in DEX
BMI (kg/m²)
Waist-to-hip-ratio
Grip strength (\%)

\begin{tabular}{c}
\hline 1st tertile, $0.5-5$ years \\
\hline $9(75)$ \\
$3.4(2.0), n=12$ \\
$52(33), n=12$ \\
$125(19), n=12$ \\
$80(14), n=12$ \\
$5.6(1.6), n=9$ \\
$198(34), n=8$ \\
$115(58), n=7$ \\
$132(127), n=8$ \\
$-1.1(2.2), n=9$ \\
$28(12), n=12$ \\
$0.9(0.2), n=6$ \\
$83(22), n=6$
\end{tabular}

\begin{tabular}{c}
\hline 2nd tertile, $5-16$ years \\
\hline $12(100)$ \\
Median (IQR), $n$ \\
$10.1(6.0), n=12$ \\
$55(32), n=12$ \\
$123(26), n=12$ \\
$78(24), n=12$ \\
$5.8(0.8), n=11$ \\
$238(62), n=11$ \\
$143(61), n=11$ \\
$212(164), n=11$ \\
$-0.9(1.8), n=8$ \\
$27(8), n=12$ \\
$0.9(0.1), n=10$ \\
$89(26), n=9$
\end{tabular}

\begin{tabular}{c}
\hline 3rd tertile, $16-50$ years \\
\hline $7(58)$ \\
$36.3(20.6), n=12$ \\
$67(22), n=12$ \\
$145(23), n=12$ \\
$90(8), n=12$ \\
$5.8(0.9), n=12$ \\
$211(62), n=12$ \\
$117(71), n=12$ \\
$121(177), n=12$ \\
$-1.4(1.5), n=12$ \\
$29(7), n=12$ \\
$1.0(0.1), n=12$ \\
$90(38), n=11$
\end{tabular}

\begin{tabular}{c}
\hline $\boldsymbol{P}$ \\
\hline \\
0.000 \\
NS \\
0.001 \\
NS \\
NS \\
NS \\
NS \\
NS \\
NS \\
NS \\
NS \\
NS
\end{tabular}

DEX, dual energy X-ray absorptiometry.

${ }^{a}$ Grip strength was assessed using the Jamar hydraulic hand dynamometer. The median values of three trials of the leading hand were compared with the median values of an age- and gender-matched normative population. 
Table 4 Rate of patients (in \%) presenting impairment in the health-related quality of life (HRQoL) compared with an age- and sexmatched healthy population.

\begin{tabular}{|c|c|c|c|c|}
\hline Instrument & $\begin{array}{l}\text { Female BADX patients } \\
(n=20) \text { compared with a } \\
\text { healthy population }(\%)\end{array}$ & $\begin{array}{l}\text { Male BADX patients }(n=6) \\
\text { compared with a healthy } \\
\text { population }(\%)\end{array}$ & $\begin{array}{l}\text { Female patients with CD } \\
(n=52)^{a} \text { compared with a } \\
\text { healthy population }(\%)\end{array}$ & $\begin{array}{l}\text { Male patients with CD } \\
(n=11)^{\mathrm{a}} \text { compared with a } \\
\text { healthy population }(\%)\end{array}$ \\
\hline SF-36 Physical functioning & 50.0 & 16.7 & & \\
\hline SF-36 Role-physical & 60.0 & 0.0 & & \\
\hline SF-36 Bodily pain & 35.0 & 16.7 & & \\
\hline SF-36 General health & 40.0 & 16.7 & & \\
\hline SF-36 Vitality & 55.0 & 16.7 & & \\
\hline SF-36 Social functioning & 55.0 & 0.0 & & \\
\hline SF-36 Role-emotional & 45.0 & 16.7 & & \\
\hline SF-36 Mental health & 35.0 & 16.7 & & \\
\hline $\begin{array}{l}\text { Tuebingen CD-25 total } \\
\text { score }\end{array}$ & 45.0 & 16.7 & 90.4 & 45.5 \\
\hline
\end{tabular}

SF-36, Short Form (36) Health Survey; CD, Cushing's disease.

a Data from the development study of the Tuebingen CD-25 (12).

patients than in males when compared with an age- and sex-matched healthy population. This can be the longterm consequence of pre-existing CS, or a result of the adrenalectomy status in the context of adrenal insufficiency. While only about $17 \%$ of male BADX patients experienced an impaired quality of life compared with the general population, about half of the female BADX patients still suffered from an impaired quality of life. However, HRQoL impairments in BADX patients of both genders were less pronounced in comparison with patients with overt CD.

The persistence of Cushing's morbidities after BADX seems to be associated with slightly higher doses of hydrocortisone used for substitution. Oversubstitution with hydrocortisone might be involved in the reappearance of hypertension. No association between mineralocorticoid substitution and blood pressure or DHEA substitution in female patients and HRQoL was found.

The incidence of 4.1 adrenal crises per 100 patientyears was relatively low compared with a median of 9.1 crises in published BADX studies (13) and with 6.3 crises in patients with Addison's disease (21). Adrenal crisis was responsible for death in only one case. By contrast, Nelson tumor occurred relatively often (24\%) compared with published data ranging from 9 to $47 \%(16,22)$. This might be due to the long follow-up period, as the median time until Nelson tumor occurrence after BADX was 51 months.

In conclusion, BADX is an effective treatment option in patients with CS. While the overall surgical procedure can be considered as relatively safe, patients who were selected for BADX suffer from clinically relevant hypercortisolism at the time of surgery, which increases the likelihood of peri-surgical complications. This has to be weighed against the considerable improvement of Cushing's symptoms, which is experienced by the majority of patients comparable to that of patients initially cured by the TSS. Long-term follow-up in specialized centers is important as some comorbidities persist and complications such as Nelson tumors can occur later after BADX.

An important limitation of the study is the retrospective data collection. However, the analysis of metabolic, cardiovascular, and psychiatric outcome parameters was performed as a standardized cross-sectional study. The ideal study to analyze the long-term follow-up of patients with CS who underwent BADX would be a multicenter registry-based study with prospective data collection.

Table 5 Median hydrocortisone substitution dose and prevalence of comorbidities and cardiovascular risk factors in patients with CS after BADX.

\begin{tabular}{l} 
Comorbidity present \\
\hline High blood pressure \\
Diabetes mellitus \\
Osteopenia or \\
osteoporosis \\
Cushing's stigmata \\
Muscle weakness \\
Psychiatric morbidity \\
Menstrual \\
irregularity
\end{tabular}

Hydrocortisone dose, median (IQR), $n$

\begin{tabular}{|c|c|c|}
\hline Yes & No & $\boldsymbol{P}$ \\
\hline 25.0 (9), 24 & 20.0 (10), 11 & 0.047 \\
\hline $27.5(8), 6$ & 25.0 (10), 29 & NS \\
\hline 25.0 (5), 24 & $20.0(10), 9$ & NS \\
\hline 25.0 (18), 8 & $25.0(5), 27$ & NS \\
\hline $25.0(15), 11$ & $25.0(8), 21$ & NS \\
\hline $25.0(10), 16$ & $25.0(5), 19$ & NS \\
\hline 22.5 (16), 4 & $25.0(15), 4$ & NS \\
\hline
\end{tabular}

${ }^{a}$ Excluding postmenopausal women. 
Declaration of interest

The authors declare that there is no conflict of interest that could be perceived as prejudicing the impartiality of the research reported.

\section{Funding}

This work has been made possible by a grant from the Else Kröner-FreseniusStiftung in support of the German Cushing's Registry CUSTODES to M Reincke (2012_A103). This work was supported financially by the German Federal Ministry of Education and Research (BMBF; m4 Cluster, 16EX1221J, $01 \mathrm{EX} 1020 \mathrm{E}$ ) and by an unrestricted educational grant from Novartis Pharma GmbH, Nürnberg, Germany. CUSTODES is part of NeoExNET (Network of Excellence for Neuroendocrine Tumors in Munich). NeoExNET is a national database for the evaluation of diagnostics, treatment, and outcomes in neuroendocrine tumors. Members of the NeoExNET-Study group include: Principal investigator, G Stalla (Max Planck Institute of Psychiatry, Munich) and Steering Committee, F Beuschlein (Ludwig Maximilian University, Munich), Christoph Auernhammer (Ludwig Maximilian University, Munich), and K A Kuhn (Technische Universität München, Munich). This study was only feasible owing to the support of our Munich Cushing's Registry team (Sabrina Hierse, Nina Nirschl and Gabriele Breu).

\section{References}

1 Feelders RA, Pulgar SJ, Kempel A \& Pereira AM. The burden of Cushing's disease: clinical and health-related quality of life aspects. European Journal of Endocrinology 2012167 311-326. (doi:10.1530/EJE-11-1095)

2 Newell-Price J, Bertagna X, Grossman AB \& Nieman LK. Cushing's syndrome. Lancet 2006367 1605-1617. (doi:10.1016/S01406736(06)68699-6)

3 Hammer GD, Tyrrell JB, Lamborn KR, Applebury CB, Hannegan ET, Bell S, Rahl R, Lu A \& Wilson CB. Transsphenoidal microsurgery for Cushing's disease: initial outcome and long-term results. Journal of Clinical Endocrinology and Metabolism 200489 6348-6357. (doi:10.1210/jc.2003-032180)

4 Invitti C, Pecori Giraldi F, de Martin M \& Cavagnini F. Diagnosis and management of Cushing's syndrome: results of an Italian multicentre study. Study Group of the Italian Society of Endocrinology on the Pathophysiology of the Hypothalamic-Pituitary-Adrenal Axis. Journal of Clinical Endocrinology and Metabolism 199984 440-448. (doi:10.1210/jcem.84.4.5629)

5 Benveniste RJ, King WA, Walsh J, Lee JS, Delman BN \& Post KD. Repeated transsphenoidal surgery to treat recurrent or residual pituitary adenoma. Journal of Neurosurgery 2005102 1004-1012. (doi:10.3171/ jns.2005.102.6.1004)

6 Jagannathan J, Sheehan JP, Pouratian N, Laws ER, Steiner L \& Vance ML. Gamma knife surgery for Cushing's disease. Journal of Neurosurgery 2007106 980-987. (doi:10.3171/jns.2007.106.6.980)

7 Petit JH, Biller BM, Yock TI, Swearingen B, Coen JJ, Chapman P, Ancukiewicz M, Bussiere M, Klibanski A \& Loeffler JS. Proton stereotactic radiotherapy for persistent adrenocorticotropin-producing adenomas. Journal of Clinical Endocrinology and Metabolism 200893 393-399. (doi:10.1210/jc.2007-1220)

8 Feelders RA \& Hofland LJ. Medical treatment of Cushing's disease. Journal of Clinical Endocrinology and Metabolism 201398 425-438. (doi:10.1210/jc.2012-3126)

9 Peters MJ, van Nes SI, Vanhoutte EK, Bakkers M, van Doorn PA, Merkies IS \& Faber CG. Revised normative values for grip strength with the Jamar dynamometer. Journal of the Peripheral Nervous System 201116 47-50. (doi:10.1111/j.1529-8027.2011.00318.x)

10 Bullinger M \& Kirchberger I. SF-36. Fragebogen zum Gesundheitszustand. Handanweisung Göttingen: Hogrefe, 1998.

11 Milian M, Teufel P, Honegger J, Gallwitz B, Schnauder G \& Psaras T. The development of the Tuebingen Cushing's disease quality of life inventory (Tuebingen CD-25). Part II: normative data from 1784 healthy people. Clinical Endocrinology 201276 861-867. (doi:10.1111/ j.1365-2265.2011.04280.x)

12 Milian M, Teufel P, Honegger J, Gallwitz B, Schnauder G \& Psaras T. The development of the Tuebingen Cushing's disease quality of life inventory (Tuebingen CD-25). Part I: construction and psychometric properties. Clinical Endocrinology 201276 851-860. (doi:10.1111/j. 1365-2265.2011.04191.x)

13 Ritzel K, Beuschlein F, Mickisch A, Osswald A, Schneider HJ, Schopohl J \& Reincke M. Outcome of bilateral adrenalectomy in Cushing's syndrome: a systematic review. Journal of Clinical Endocrinology and Metabolism 201398 3939-3948. (doi:10.1210/jc. 2013-1470)

14 Acosta E, Pantoja JP, Gamino R, Rull JA \& Herrera MF. Laparoscopic versus open adrenalectomy in Cushing's syndrome and disease. Surgery 1999126 1111-1116. (doi:10.1067/msy.2099.102423)

15 Lindholm J, Juul S, Jorgensen JO, Astrup J, Bjerre P, Feldt-Rasmussen U, Hagen C, Jorgensen J, Kosteljanetz M, Kristensen L et al. Incidence and late prognosis of Cushing's syndrome: a population-based study. Journal of Clinical Endocrinology and Metabolism 200186 117-123.

16 Assie G, Bahurel H, Coste J, Silvera S, Kujas M, Dugue MA, Karray F, Dousset B, Bertherat J, Legmann P et al. Corticotroph tumor progression after adrenalectomy in Cushing's disease: a reappraisal of Nelson's syndrome. Journal of Clinical Endocrinology and Metabolism 200792 172-179. (doi:10.1210/jc.2006-1328)

17 Pereira AM, Tiemensma J \& Romijn JA. Neuropsychiatric disorders in Cushing's syndrome. Neuroendocrinology 201092 (Suppl 1) 65-70. (doi:10.1159/000314317)

18 Chow JT, Thompson GB, Grant CS, Farley DR, Richards ML \& Young WF Jr. Bilateral laparoscopic adrenalectomy for corticotrophindependent Cushing's syndrome: a review of the Mayo Clinic experience. Clinical Endocrinology 200868 513-519. (doi:10.1111/j. 1365-2265.2007.03082.x)

19 Colao A, Pivonello R, Spiezia S, Faggiano A, Ferone D, Filippella M, Marzullo P, Cerbone G, Siciliani M \& Lombardi G. Persistence of increased cardiovascular risk in patients with Cushing's disease after five years of successful cure. Journal of Clinical Endocrinology and Metabolism 199984 2664-2672. (doi:10.1210/jcem.84.11.6112)

20 Schneider HJ, Friedrich N, Klotsche J, Pieper L, Nauck M, John U, Dorr M, Felix S, Lehnert H, Pittrow D et al. The predictive value of different measures of obesity for incident cardiovascular events and mortality. Journal of Clinical Endocrinology and Metabolism 201095 1777-1785. (doi:10.1210/jc.2009-1584)

21 Hahner S, Loeffler M, Bleicken B, Drechsler C, Milovanovic D, Fassnacht M, Ventz M, Quinkler M \& Allolio B. Epidemiology of adrenal crisis in chronic adrenal insufficiency: the need for new prevention strategies. European Journal of Endocrinology 2010162 597-602. (doi:10.1530/EJE-09-0884)

22 Thompson SK, Hayman AV, Ludlam WH, Deveney CW, Loriaux DL \& Sheppard BC. Improved quality of life after bilateral laparoscopic adrenalectomy for Cushing's disease: a 10-year experience. Annals of Surgery 2007245 790-794. (doi:10.1097/01.sla. $0000251578.03883 .2 \mathrm{f}$ ) 